\title{
Treatment of high- and intermediate-risk pulmonary embolism using the AngioJet percutaneous mechanical thrombectomy system in patients with contraindications for thrombolytic treatment - a pilot study
}

\author{
Paweł Latacz ${ }^{1}$, Marian Simka², Pawel Brzegowy³, Wojciech Serednicki ${ }^{4}$, Ewa Konduracka ${ }^{5}$, Wojciech Mrowiecki ${ }^{6}$, \\ Agnieszka Słowik ${ }^{1}$, Bartłomiej Łasocha ${ }^{7}$, Tomasz Mrowiecki ${ }^{6}$, Tadeusz Popiela ${ }^{3}$ \\ 1Department of Neurology, Jagiellonian University Medical College, Krakow, Poland \\ ${ }^{2}$ Department of Anatomy, University of Opole, Poland \\ ${ }^{3}$ Chair of Radiology, Jagiellonian University Medical College, Krakow, Poland \\ ${ }^{4}$ Department of Anaesthesiology and Intensive Care Unit, Jagiellonian University Medical College, University Hospital, Krakow, Poland \\ ${ }^{5}$ Coronary Disease and Heart Failure Department, Jagiellonian University Medical College, John Paul II Hospital, Krakow, Poland \\ ${ }^{6}$ Department of Vascular Surgery, University Hospital, Krakow, Poland \\ ${ }^{7}$ Diagnostic Imaging Center of Rescue Medicine and Multiorgan Trauma Department, University Hospital, Krakow, Poland
}

Videosurgery Miniinv 2018; 13 (2): 233-242

DOI: https://doi.org/10.5114/wiitm.2018.75848

\begin{abstract}
Introduction: Intravenous thrombolysis is the treatment of choice in patients presenting with high-and intermediate-risk pulmonary embolism. The role of percutaneous mechanical pulmonary thrombectomy (PMPT) is not fully established, although selected patients can be managed with this method.

Aim: This open-label single-centre prospective pilot study was aimed at assessing the feasibility of PMPT for the treatment of severe pulmonary embolism in a Polish hospital. We also evaluated the safety and efficacy of such management.

Material and methods: We managed 7 patients, aged $52.7 \pm 16.6$ years, presenting with high-and intermediate-risk pulmonary embolism (4 patients with class 5 and one patient with class 4 of the Pulmonary Embolism Severity Index), with occlusion of at least 2 lobar arteries and contraindications for thrombolysis. Percutaneous mechanical pulmonary thrombectomy was performed using the Angiojet system.

Results: It was possible to introduce the thrombectomy system to the pulmonary arteries in all patients. The procedure was successful in 6 patients (technical success rate: 85.7\%). Two (28.6\%) patients died during the hospital stay, one patient with unsuccessful thrombectomy and the other due to pneumonia. In all survivors control echocardiography demonstrated normalised function of the right ventricle. Also, dyspnoea disappeared and blood gas parameters normalised. There was no recurrent thromboembolism during 3-14 months of follow-up.

Conclusions: In the Polish setting, in selected patients, management of high-and intermediate-risk pulmonary embolism with PMPT is technically feasible. Such treatment is relatively safe and effective. It can be an alternative to standard management, especially in patients with contraindications for fibrinolysis or surgical embolectomy.
\end{abstract}

Key words: thromboembolic disease, pulmonary embolism, mechanical thrombectomy system, pulmonary artery. 


\section{Introduction}

Venous thromboembolic disease represents the third most common cause of cardiovascular death in the United States and Europe [1-3]. Symptomatic pulmonary embolism (PE) occurs in about 500,000 patients annually, with an estimated mortality as high as $30 \%$ in high-risk patients [2, 3]. According to current guidelines, based on clinical and haemodynamic criteria and the risk of early mortality, severe PE can be categorised into two main groups [2-5]. The first one comprises high-risk PE, which clinically manifests with haemodynamic instability and systemic hypotension (systolic blood pressure < $90 \mathrm{~mm} \mathrm{Hg}$, pressure drop of more than $40 \mathrm{~mm} \mathrm{Hg}$ or requiring administration of inotropic agents $[5,6])$. In this form of PE imaging studies usually reveal a "saddle embolus" at the bifurcation of the pulmonary trunk, embolism of the main pulmonary artery, or embolic occlusion of at least two lobar arteries [3-6]. Mortality in high-risk PE is at the level of $60 \%$, and in $66 \%$ of these patients fatal outcomes take place during the first hours from the onset of clinical symptoms [3-5]. The remaining patients with severe $\mathrm{PE}$, those with intermediate risk of mortality, do not reveal hypotension, but present with clinical symptoms comprising dyspnoea and/or tachycardia. Laboratory diagnostics, ECG and imaging studies demonstrate in these patients significant overload of the right ventricle, severe blood gas disturbances and in some patients also markers of myocardial necrosis. Estimated 30-day mortality in patients with intermediate risk PE is at the level of $15-20 \%$, and these patients are at a risk of developing pulmonary hypertension and right ventricle heart failure [7-9].

Pulmonary embolism is primarily managed with anticoagulants and fibrinolytic agents, while surgical pulmonary embolectomy is performed in selected patients [5, 7-11]. Given the fact that as many as $40 \%$ of patients with class 4 or 5 of the Pulmonary Embolism Severity Index (PESI) present with contraindications for both surgical embolectomy and fibrinolytic therapy, percutaneous mechanical pulmonary thrombectomy (PMPT) seems to be an attractive alternative treatment modality [6, 10, 12-17]. Recently a number of studies on the use of PMPT for the management of PE in patients presenting with high or intermediate risk have been published. They primarily comprised open-label studies and retrospective analyses of the cohorts, but also a few pro- spective trials. Still, to the best of our knowledge, a similar paper on Polish patients has not yet been published.

\section{Aim}

This open-label study was aimed at assessing the feasibility of management of severe PE using PMPT in a Polish hospital. We also evaluated the safety and efficacy of such treatment, with reference to the results of this treatment in other countries [6, 10, 12-18].

\section{Material and methods}

In 2015 the University Hospital in Krakow initiated a pilot programme of the management of patients presenting with high- and intermediate-risk PE, 4 or 5 class of PESI, dysfunction of the right ventricle, elevated troponin and contraindications for fibrinolytic therapy. Absolute contraindications for fibrinolysis comprised head trauma, brain surgery and other major surgical procedures. Relative contraindications regarded patients with traumatic and surgical injuries associated with a high risk of uncontrollable bleeding, such as recent fracture or patients after cardiopulmonary resuscitation with injuries of the ribs and sternum, and patients with neurological pathologies that potentially could be exacerbated by bleeding to the nervous tissue, such as polyneuropathy. Although several endovascular techniques can be used for the purpose of mechanical thrombectomy of the pulmonary arteries, considering the fact that our team has expertise in the use of the AngioJet (Boston Scientific, Natick, MA, USA) rheolytic thrombectomy system, we decided to use this endovascular device. For the purpose of this programme and also for everyday practice, we created an algorithm - how to diagnose and manage PE patients, and which patients should be qualified for PMPT (Figure 1). The study protocol has been approved by the Bioethical Committee of the Regional Board of Physicians in Krakow (approval N ${ }^{0} 138 / \mathrm{KBL} /$ OIL/2015).

We primarily focused at the feasibility of PMPT, which was defined as the rate of technically successful procedures, and at safety of such management measured by in-hospital mortality and prevalence of severe adverse events. We also performed an analysis of how PMPT improved haemodynamic, angiographic and clinical parameters in these patients. 


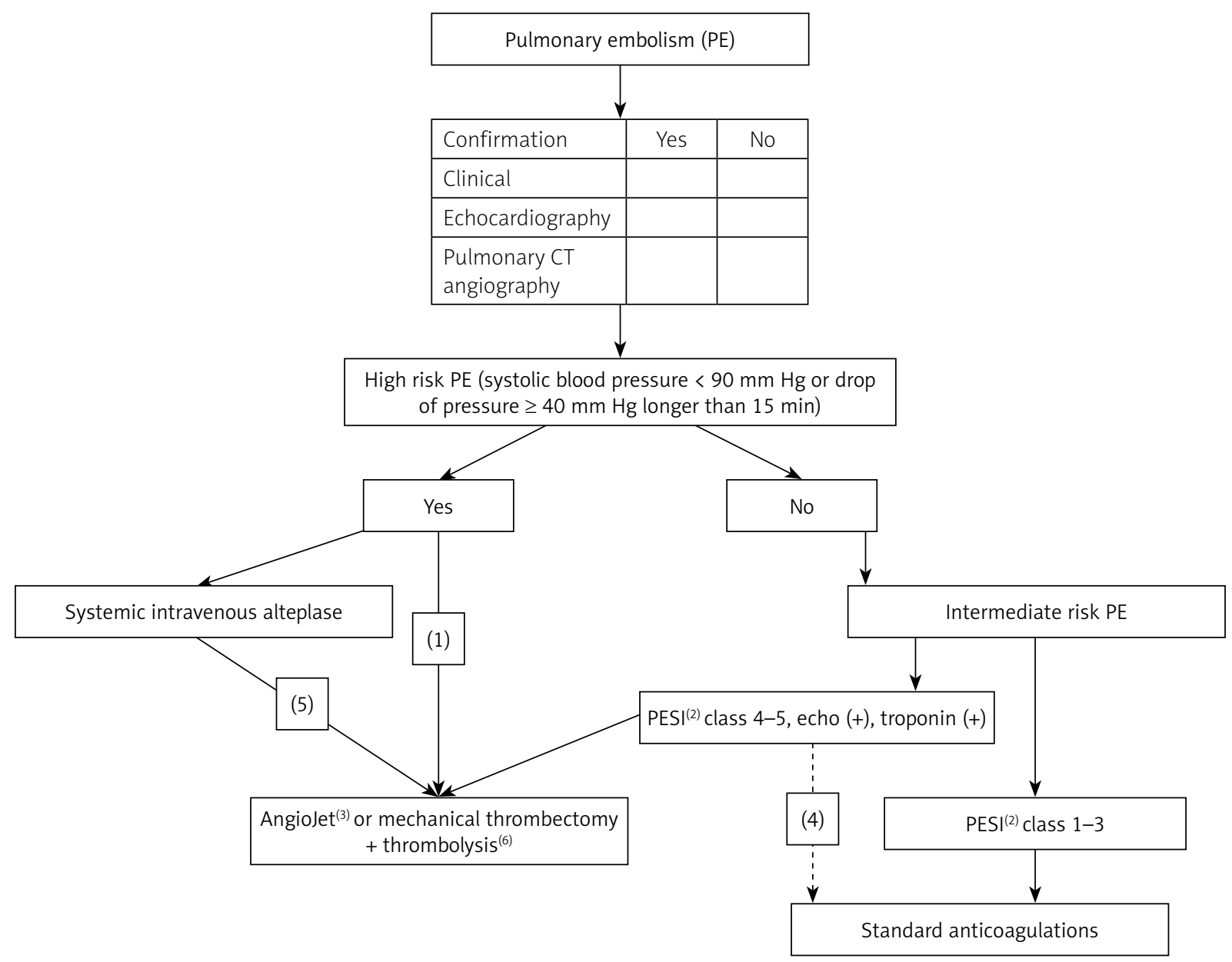

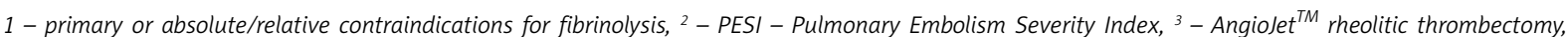

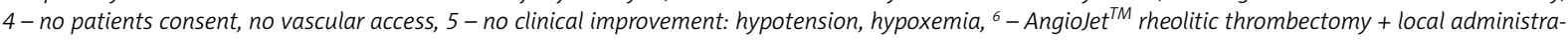
tion of alteplase.

Figure 1. Algorithm of management of patients with pulmonary embolism in our hospital

Analysed clinical criteria comprised improvement of function of the right ventricle, postprocedural adverse events and in-hospital mortality. Haemodynamic and laboratory parameters, such as systolic blood pressure, heart rate and blood gases parameters ( $\mathrm{pH}$ and $\mathrm{O}_{2}$ saturation) were evaluated before and after the procedure (Table I). Echocardiography was performed before endovascular thrombectomy, $6-12 \mathrm{~h}$ after completion of PMPT and on the $2^{\text {nd }}-4^{\text {th }}$ postprocedural day.

We prospectively evaluated results of the treatment in 7 patients ( 4 women and 3 men) who were managed from December 2015 to April 2017. Patients were aged $52.7 \pm 16.6$ years. All of them presented with high or intermediate risk PE, and the diagnosis was confirmed by computed tomography (CT) angiography (Tables II and III). There were also
4 patients who met the above-described criteria but for different reasons were not managed with PMPT (Figure 2). Out of these patients, 3 of them improved clinically during preparation for the procedure and no longer presented with a high risk class according to the PESI. Another patient, with a long history of PE and haemodynamic instability, was managed with thrombolysis instead of PMPT, since considering the duration of the embolism and poor clinical status of this individual, the endovascular approach seemed technically challenging and very risky.

According to our protocol (Figure 1), patients presenting with high- or intermediate-risk PE, 4 and 5 classes PESI, with occlusion of at least 2 lobar arteries and contraindications for thrombolysis were managed with PMPT. Other patients received standard treatment using thrombolysis or antithrom- 


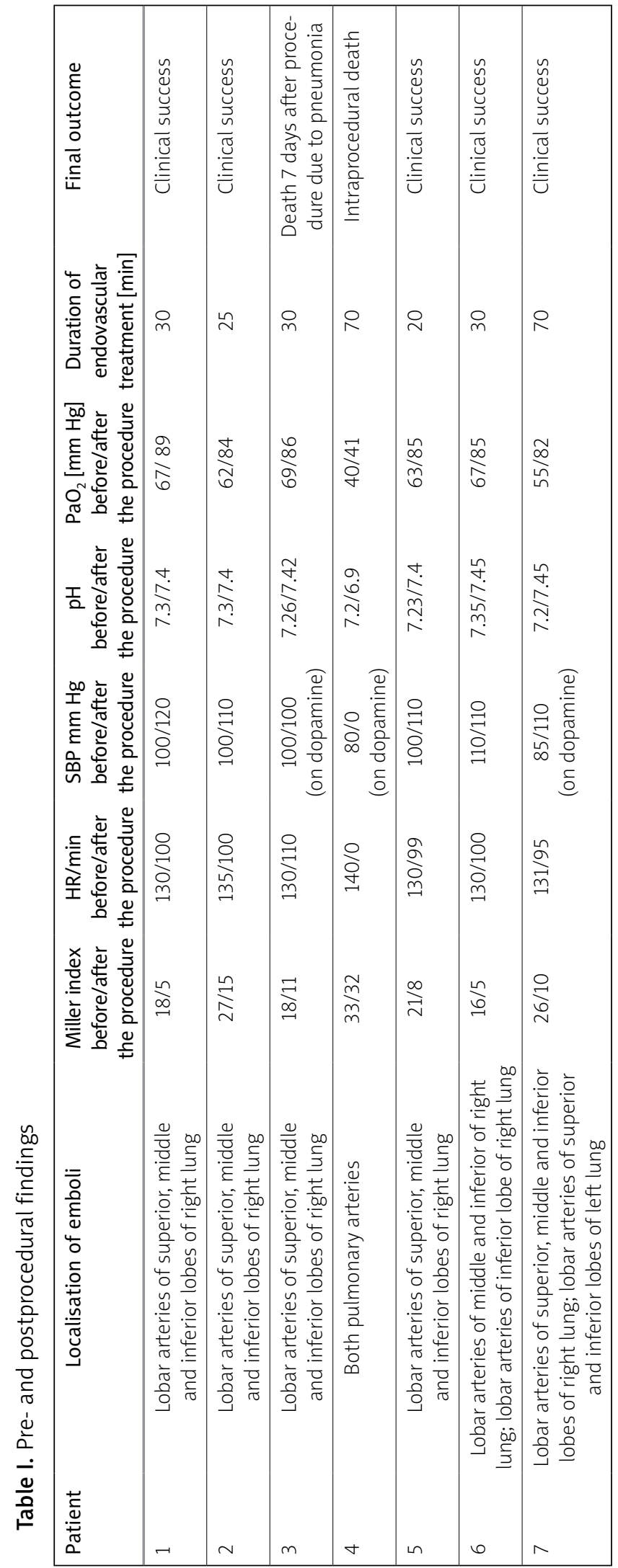

botic agents. Out of these 7 patients managed with PMPT, there was one presenting with PESI class 4 and 4 patients with PESI class 5 . Four patients provided their written informed consent to undergo the procedure, while the remaining 3 patients were unconscious and thrombectomy was performed as a life-saving intervention. Three patients required mechanical ventilation before and during the endovascular procedure. There were cardiac arrests in 2 patients before the procedure and in 1 patient during the procedure.

All patients managed with PMPT were administered therapeutic doses of low-molecular-weight heparin once PE has been suspected. In the case of relative contraindications for anticoagulation (there was 1 patient after head trauma and 2 patients after abdominal surgery) patients received subtherapeutic doses of heparin. In addition, in 2 patients with contraindications for therapeutic anticoagulation we implanted retrievable cava filters. After PMPT patients were managed in the intensive care unit. Once their clinical status became stable, which occurred on the $3^{\text {rd }}-13^{\text {th }}$ postprocedural day, they were transferred to the internal medicine ward and after further stabilisation of their status they were discharged home, with the recommendation of longterm oral anticoagulation.

\section{Endovascular technique}

In this group of patients we did not use mechanical thrombectomy augmented by local fibrinolysis, which was an option in our protocol (Figure 1). Endovascular thrombectomy was performed 3-20 h from the onset of symptoms of PE (Table III). Femoral access was the preferred route for this intervention. This vein was cannulated with a $5 \mathrm{Fr}$ or $6 \mathrm{Fr}$ introducer sheath. Then, over a standard (such as InQwire Guide Wire, Merit Medical Systems) or hydrophilic (for example, AqWire, Covidien, ev3 Endovascular, Inc., Plymouth, MN, USA) guide wire, a pigtail diagnostic catheter was positioned in the pulmonary trunk. This diagnostic catheter was then advanced into each pulmonary artery and angiography was performed. Routinely we used $10 \mathrm{ml}$ of contrast on each side, or $30 \mathrm{ml}$ in the case of injection of contrast at the level of the pulmonary trunk. This volume was adjusted depending on the clinical status of the patient and the value of the pulmonary pressure. In the case of poor clinical status of the patient 
and/or echocardiographic features of right ventricle overload, we tried to reduce the volume of contrast to 8-10 ml. After confirmation of embolisation, the above-described introducer sheath was replaced with a bigger one (6 $\mathrm{Fr}$ or $7 \mathrm{Fr}$ ). We suggest using the SiteSeer Judkins Right catheter (Medtronic, Minneapolis, MN, USA) or a long sheath with a special tip, such as the $6 \mathrm{Fr} 90 \mathrm{~cm}$ length Destination Guiding Sheath (Terumo, Tokyo, Japan). Then the AngioJet system was introduced. Using this device emboli were subsequently aspired from all occluded pulmonary branches. Initially we performed 2-5 passages of the thrombectomy device in the occluded artery, with simultaneous assessment of the tolerance of the intervention by the patient. Then we evaluated the degree of residual stenosis in angiography. Of note, we did not perform embolectomy of small branches of the pulmonary artery, since navigation through these tiny vessels is associated with very high risk of haemorrhage. We also avoided aspirations of embolic material lasting longer than 7-10 s, since such manoeuvres can result in dyspnoea and cardiac decompensation. Thrombectomy was continued until all major branches of the pulmonary arteries became recanalised, or the patient significantly improved clinically in terms of haemodynam-

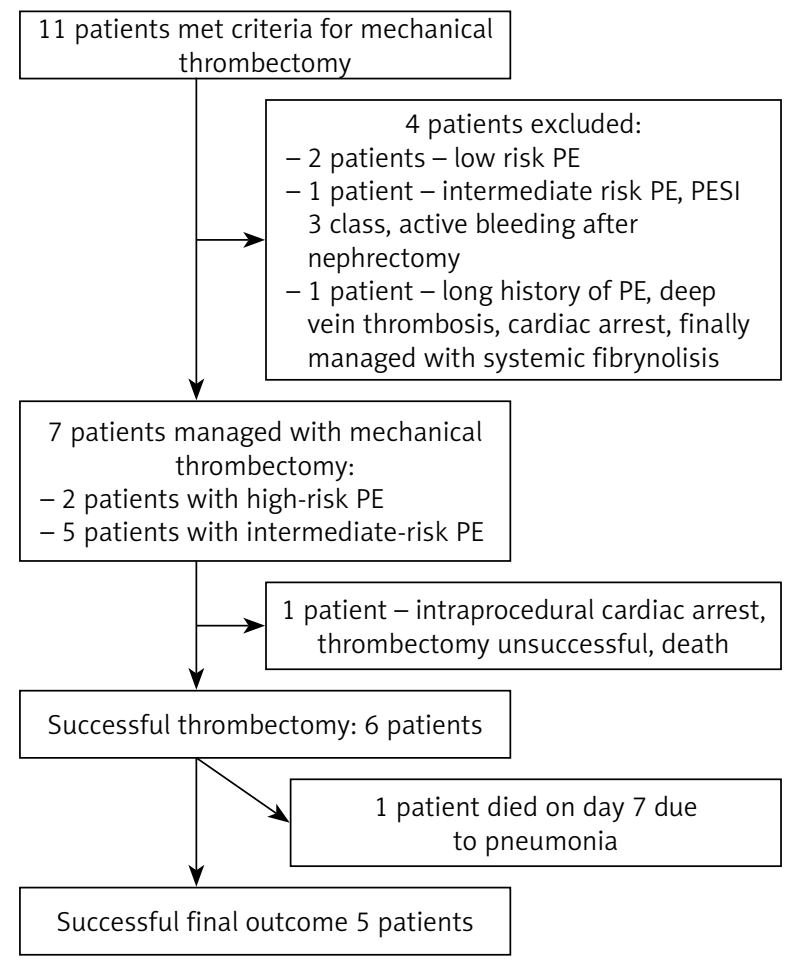

Figure 2. Flow chart of patients
Table II. Demographic, clinical and diagnostic data of all treated patients $(n=7)$

\begin{tabular}{|c|c|}
\hline Parameter & Value \\
\hline \multicolumn{2}{|l|}{ Gender: } \\
\hline Males & $3(43 \%)$ \\
\hline Females & $4(57 \%)$ \\
\hline Age, mean \pm SD [years] & $52.7 \pm 16.6$ \\
\hline \multicolumn{2}{|l|}{ Risk factors for PE: } \\
\hline Immobilisation & $2(29 \%)$ \\
\hline Recent surgery & $2(29 \%)$ \\
\hline Malignancy & $1(14 \%)$ \\
\hline Recent trauma & $1(14 \%)$ \\
\hline Obesity & $1(14 \%)$ \\
\hline Contraindications for thrombolysis & $7(100 \%)$ \\
\hline \multicolumn{2}{|l|}{ Symptoms: } \\
\hline Dyspnoea & $7(100 \%)$ \\
\hline Chest pain & $4(57 \%)$ \\
\hline Presyncope/syncope & $3(43 \%)$ \\
\hline Cardiac arrest & $2(29 \%)$ \\
\hline Palpitations & $2(29 \%)$ \\
\hline \multicolumn{2}{|l|}{ Clinical presentation: } \\
\hline Intermediate-risk pulmonary embolism: & $5(71 \%)$ \\
\hline PESI class 5 & $4(57 \%)$ \\
\hline PESI class 4 & $1(14 \%)$ \\
\hline High-risk pulmonary embolism + shock & $2(43 \%)$ \\
\hline Hypoxia & $7(100 \%)$ \\
\hline Tachycardia (heart rate > 130/min) & $7(100 \%)$ \\
\hline \multicolumn{2}{|l|}{ Diagnostic modalities: } \\
\hline Right heart strain & $7(100 \%)$ \\
\hline Right ventricular dilatation & $6(86 \%)$ \\
\hline Troponin I > $0.01 \mathrm{ng} / \mathrm{ml}$ & $6(86 \%)$ \\
\hline Right ventricle failure (echocardiography) & $7(100 \%)$ \\
\hline Paradoxical motion of interventricular septum & $2(29 \%)$ \\
\hline $\begin{array}{l}\text { Embolism of both pulmonary arteries in } \mathrm{CT} \\
\text { angiography }\end{array}$ & $1(14 \%)$ \\
\hline $\begin{array}{l}\text { More than } 2 \text { lobar arteries occluded in CT } \\
\text { angiography }\end{array}$ & $7(100 \%)$ \\
\hline $\begin{array}{l}\text { Deep vein thrombosis of the lower extremities } \\
\text { demonstrated by sonographic examination }\end{array}$ & $2(29 \%)$ \\
\hline
\end{tabular}




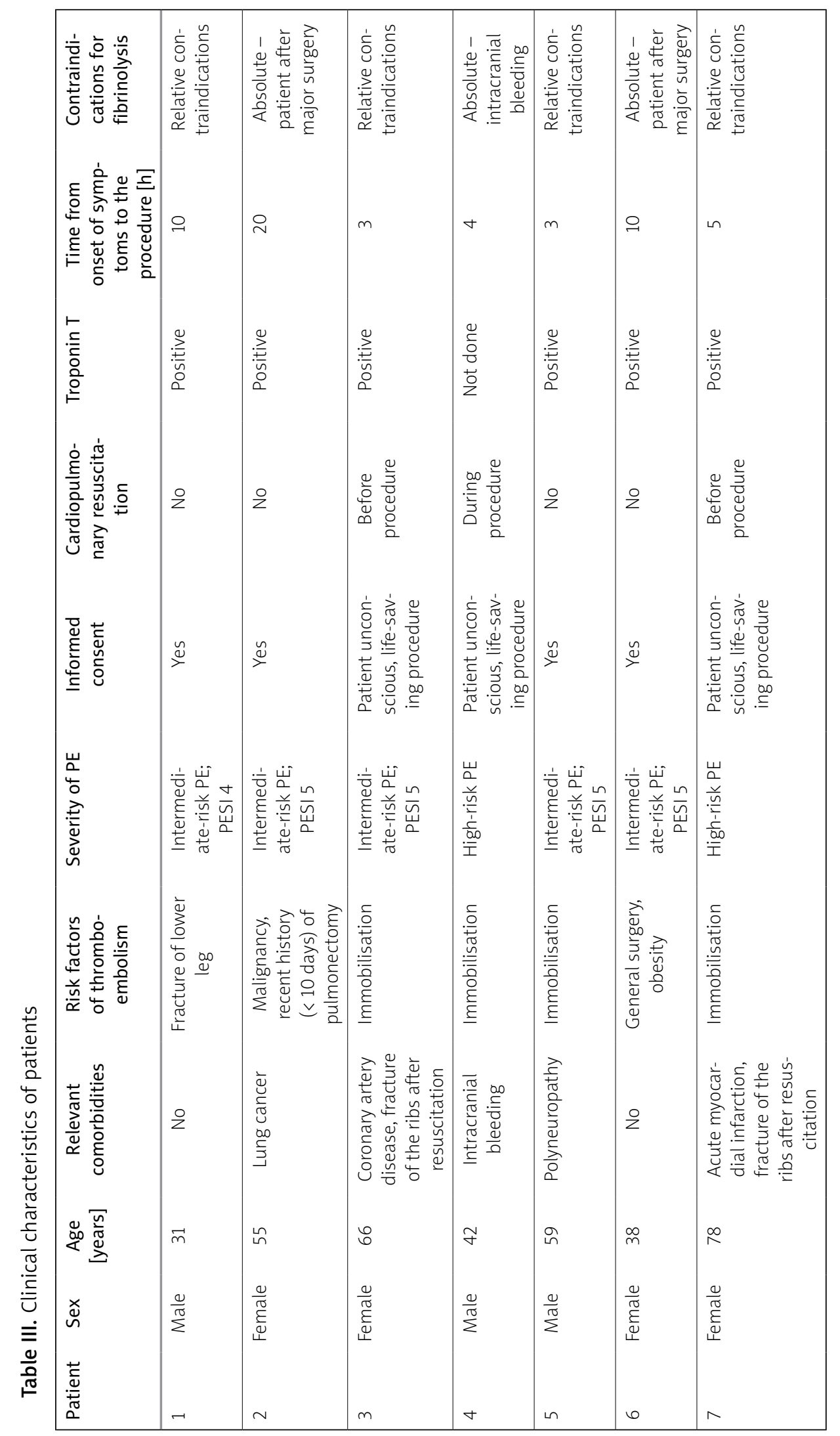


ic stability and blood gas parameters, even if the angiographic result was suboptimal. We regarded a $30 \%$ residual stenosis as acceptable on condition that there was good inflow to peripheral branches of the pulmonary arteries. Also $30-50 \%$ stenosis with significant improvement of clinical status (normalisation of heart rate and blood gas parameters) and good inflow to peripheral branches was interpreted as a good result. Residual stenosis more than $50 \%$ or no clinical improvement represented an indication for more passages of the thrombectomy system with subsequent angiographic control. In this patient series in each individual we performed 3-6 aspirations with the AngioJet system. Duration of the procedure ranged from 30 to $70 \mathrm{~min}$ and the total volume of contrast injected ranged from 50 to $100 \mathrm{ml}$.

\section{Statistical analysis}

Statistical analysis was performed using the SigmaStat for Mac, Version 7.0 software (SPSS Inc., Chicago, IL, USA). Continuous variables were expressed as medians, categorical data as numbers and percentages.

\section{Results}

Diagnosis of PE was made based on the results of pulmonary CT angiography. CT angiography in 1 patient revealed an occlusion of both pulmonary arteries, in 5 patients occlusion of three lobar arteries and in 1 patient occlusion of five lobar arteries (Table I). The Miller index (an angiographic measure of pulmonary occlusion, which is calculated as the sum of obstruction and perfusion indexes, ranging from $0=$ best to $34=$ worst) scored 16-33 (Table I). In addition, in all patients echocardiography demonstrated signs of right ventricle overload, such as dilatation of the right ventricle (7 patients) and abnormal mobility of the interventricular septum (3 patients).

In total, 2 patients died during the hospital stay, 1 patient with intraprocedural cardiac arrest and unsuccessful thrombectomy (mentioned above), and another patient who died 7 days after the procedure due to pneumonia. Thus, the in-hospital mortality was $28.6 \%$. Except for 1 case of intraprocedural death and 1 case of fatal pneumonia, there were no serious adverse events directly associated with PMPT, such as perforation of the pulmonary artery or another blood vessel, distal embolisation or bleeding from the access site. In 3 individuals, who were our first patients managed with PMPT, there were bradyarrhythmias during a longer aspiration of embolic material. These symptoms ceased spontaneously with discontinuation of aspiration; thus we consider these adverse events as moderate ones. Based on these experiences we limited the time of aspiration to 7-10 s, and bradyarrhythmias were not seen in subsequent patients.

It was possible to introduce the Angiolet system to pulmonary arteries in all patients. Mechanical thrombectomy with this device was successful in 6 patients (technical success rate $-85.7 \%$ ). The only patient with unsuccessful thrombectomy died during the procedure.

Five PMPT procedures were successful (example results of thrombectomy - Photos 1 and 2). In these patients normalisation of heart rate took place during the first $2 \mathrm{~h}$ after the procedure, while the improvement of respiratory function manifesting with disappearance of dyspnoea and normalisation of blood gas parameters occurred 2-6 h after thrombectomy. Only 1 patient, with a recent history of myocardial infarction, required a 3-days long infusion of dopamine. The remaining 4 patients were haemodynamically stable just after the procedure. Control echocardiography in all survivors demonstrated normalised function of the right ventricle.

Duration of hospital stay in survivors was $6-20$ days. There were no recurrent thromboembolic events during 3-14 months of follow-up. Changes of angiographic and laboratory parameters after thrombectomy are summarised in Table I.

\section{Discussion}

The results of our case series suggest that PMPT can be seen as an alternative to standard management, especially in patients with contraindications for fibrinolysis or surgical embolectomy. A small sample size, single-centre study and no control group are the main limitations of this survey. On the other hand, a standardised protocol for PMPT seems to be a strong point of our report.

Early aggressive treatment restoring patency of occluded pulmonary arteries represents the principal factor affecting mortality in severe PE [2]. According to the guidelines of the European Society of Cardiology, shock or systemic hypotension is an accepted indication for urgent thrombolysis in patients with acute PE $[2,7]$. Surgical or endovascular 

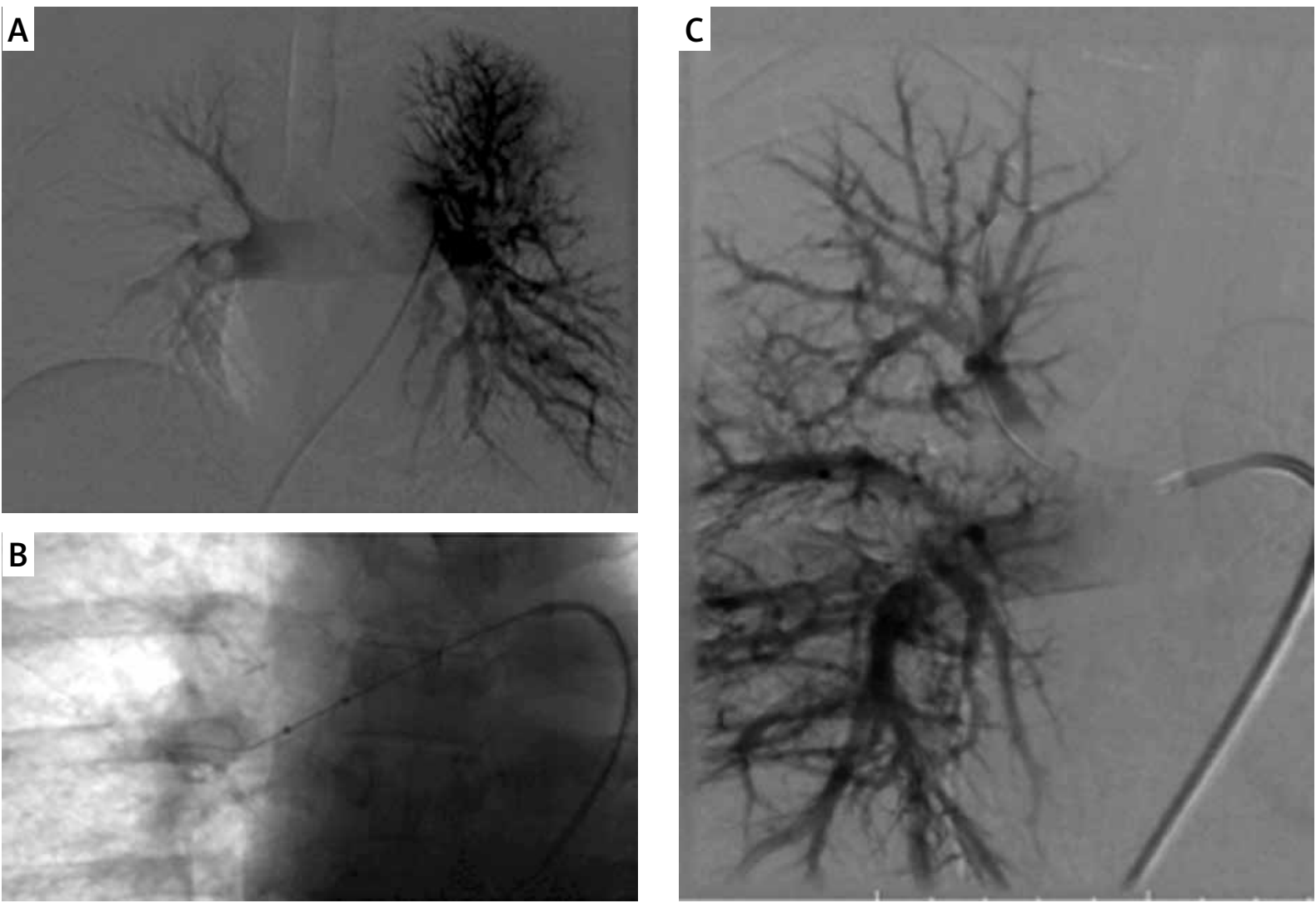

Photo 1. Result of thrombectomy in patient with right-sided pulmonary embolism: A - initial image - occlusion of the middle and inferior lobar arteries, and also partial occlusion of the superior lobar artery, B - Angiojet system introduced to the pulmonary artery, $\mathbf{C}$ - final result

thrombectomy can be an option in selected cases [10, 12-17]. Although intravenous systemic thrombolysis is recommended as a life-saving treatment in severe PE, the clinical benefit of such management is not obvious. According to the ICOPER registry, systemic fibrinolysis did not affect mortality in patients presenting with high-risk PE [3]. The American College of Chest Physicians recommends an endovascular intervention only in patients with contraindications for fibrinolysis, primarily those with a high risk of bleeding [19], while the European Society of Cardiology suggests the endovascular approach as an alternative for surgical embolectomy in patients with unsuccessful thrombolysis [7]. Still, a number of single-centre studies, as well as meta-analyses of such trials, have demonstrated that PMPT in high- or intermediate risk-PE can be safe and effective, and that the risk of serious adverse events is comparable or even lower than that after fibrinolytic therapy [6, 10, 12-17]. This relatively high efficacy of PMPT is related to the fact that mechanical fragmentation of the embolus increases the chance of recanalisation of the pulmonary artery. In addition, the dose of fibrinolytic agent that is directly administered to the pulmonary arteries is reduced and therefore bleeding complications are less frequent [3, 6, 14-16]. Consequently, in some highly experienced centres PMPT is regarded as the procedure of choice, even if systemic thrombolysis and surgical embolectomy still play important roles [6, 10, 13-17]. It has been emphasized that clinical success of PMPT is primarily associated with recanalisation of pulmonary and/ or lobar arteries. This in line with our observations. In our material intraprocedural death occurred in a patient with unsuccessful thrombectomy of the pulmonary arteries. Also, following observations of other researchers who demonstrated that the decision upon completion of the procedure should be based on the clinical and not angiographic outcome, we finished PMPT once the patient clinically improved, irrespective of the angiographic picture of the pulmonary circulation. Such a strategy is due 

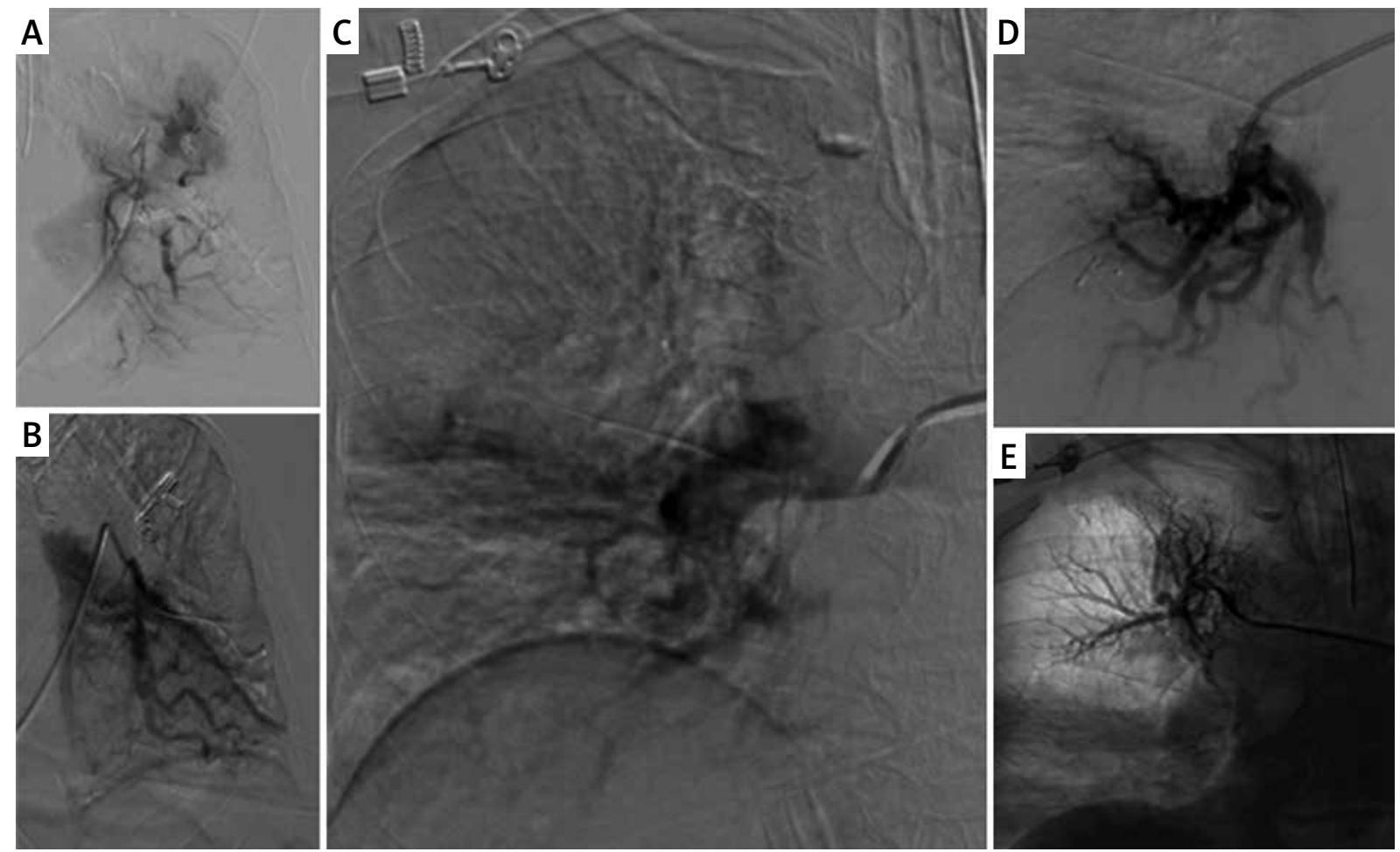

Photo 2. Result of thrombectomy in patient with bilateral occlusion of pulmonary arteries: A - initial image: occlusion of the left superior and inferior lobar arteries, $\mathbf{B}$ - after thrombectomy of the inferior lobar arteries of the left lung, $\mathbf{C}$ - occlusion of the superior, middle and inferior lobar arteries of the right lung, D - recanalisation of the inferior, $\mathrm{E}$ - superior lobar arteries

to the fact that thrombectomy of distal pulmonary vessels is associated with especially high risk of life-threatening technical failures $[6,10,15]$.

The question whether PMPT should be followed by local pharmacological thrombolysis is also under debate. Lee et al. reported the results of such management in 91 patients presenting with intermediate risk PE. They administered alteplase, with mean time of drug infusion $18 \mathrm{~h}$, and observed clinical improvements in all patients [20]. This observation suggests that low-dose local thrombolysis can be an effective additional treatment in patients without contraindications for fibrinolytics. There were published two studies assessing long-term local fibrinolysis using the EkoSonic Endovascular System [21, 22]. The system has been found effective in patients presenting with intermediate-risk acute PE and the results were superior to standard anticoagulation with heparin. In the first study the authors used 10-20 mg of alteplase, while in the other the total dose of alteplase was $24 \mathrm{mg}$. Consequently, there were significantly different rates of hemorrhagic adverse events (0\% vs. $10 \%)$. It should also be emphasized that in the first study [21] $84 \%$ of patients did not meet the inclusion criteria; thus the group assessed was very highly selected.

Bradyarrhythmia associated with longer aspirations of embolic material is a well-known problem. This complication was seen in $15 \%$ of patients managed by some authors [23-25], while others did not report these adverse events [6, 10]. Short aspirations seem to be the best measure to avoid these complications. In is not clear which factors trigger bradyarrhythmias. Some authors blamed haemolysis-related hyperkalaemia, adenosine or a spasm of arteries caused by nitric oxide sequestrated in the pulmonary circulation [24, 25]. We did not use prophylaxis with aminophylline, which was recommended by some authors [25]. Also, in our patients management of bradyarrhythmias with endocavitary stimulation was not needed.

Some authors reported quite a high rate of serious adverse events associated with the use of the Angiolet system, such as haemoptysis, renal failure, peripheral embolisation and bradyarrhythmias [23]. Still others 
Paweł Latacz, Marian Simka, Pawel Brzegowy, Wojciech Serednicki, Ewa Konduracka, Wojciech Mrowiecki, Agnieszka Słowik, Bartłomiej Łasocha, Tomasz Mrowiecki, Tadeusz Popiela

found this endovascular device to be rather safe [6, 10]. In our patients, except for bradyarrhythmias, there were no serious complications, and even those could be avoided after modification of the procedure.

\section{Conclusions}

The results of treatment of our patient series, even respecting the fact that this was a small group, demonstrates that in the Polish context PMPT of high- or intermediate-risk PE is technically feasible, and such management is relatively safe and effective.

\section{Conflict of interest}

\section{The authors declare no conflict of interest.}

\section{References}

1. Tapson VF. Acute pulmonary embolism. N Engl J Med 2008; 358: 1037-52.

2. Cohen AT, Agnelli G, Anderson FA, et al. VTE Impact Assessment Group in Europe (VITAE), Venous thromboembolism (VTE) in Europe. The number of VTE events and associated morbidity and mortality. Thromb Haemost 2007; 98: 756-64.

3. Goldhaber SZ, Visani L, De Rosa M. Acute pulmonary embolism: clinical outcomes in the International Cooperative Pulmonary Embolism Registry (ICOPER). Lancet 1999; 353: 1386-9.

4. Heit JA, Cohen AT, Anderson FA. Estimated annual numbers of US acute-care hospital patients at risk for venous thromboembolism. Am J Hematol 2007; 82: 777-82.

5. Agnelli G, Becattini C. Acute pulmonary embolism. N Engl I Med 2010; 363: 266-74.

6. Nassiri N, Jain A, McPhee D, et al. Massive and submassive pulmonary embolism: experience with an algorithm for catheter-directed mechanical thrombectomy. Ann Vasc Surg 2012; 26: 18-24.

7. Konstantinides SV, Torbicki A, Agnelli G, et al. Task Force for the Diagnosis and Management of Acute Pulmonary Embolism of the European Society of Cardiology (ESC), 2014 ESC guidelines on the diagnosis and management of acute pulmonary embolism. Eur Heart J 2014; 35: 3033-69.

8. Casazza F, Becattini C, Bongarzoni A, et al. Clinical features and short term outcomes of patients with acute pulmonary embolism. The Italian Pulmonary Embolism Registry (IPER). Thromb Res 2012; 130: 847-52.

9. Piazza G, Goldhaber SZ. Chronic thromboembolic pulmonary hypertension. N Engl J Med 2011; 364: 351-60.

10. Chechi T, Vecchio S, Spaziani G, et al. Rheolytic thrombectomy in patients with massive and submassive acute pulmonary embolism. Catheter Cardiovasc Interv 2009; 73: 506-13.

11. Lehnert P, Møller CH, Mortensen J, et al. Surgical embolectomy compared to thrombolysis in acute pulmonary embolism: morbidity and mortality. Eur J Cardiothor Surg 2017; 51: 354-61.

12. Latacz P, Simka M, Ludyga T, et al. Endovascular thrombectomy with the Angiolet system for the treatment of intermedi- ate-risk acute pulmonary embolism: a case report of two patients. Postep Kardiol Inter 2016; 12: 61-4.

13. Margheri M, Vittori G, Vecchio S, et al. Early and long-term clinical results of AngioJet rheolytic thrombectomy in patients with acute pulmonary embolism. Am J Cardiol 2008; 101: 252-8.

14. Arzamendi D, Bilodeau L, Ibrahim R, et al. Role of rheolytic thrombectomy in massive pulmonary embolism with contraindication to systemic thrombolytic therapy. Eurolntervention 2010; 5: 716-21.

15. Jain A, McPhee D, Mina B, et al. Massive and submassive pulmonary embolism: experience with an algorithm for catheter-directed mechanical thrombectomy. Ann Vasc Surg 2012; 26: 18-24.

16. Bajaj N, Kalra R, Arora P, et al. Catheter-directed treatment for acute pulmonary embolism: systematic review and single-arm meta-analyses. Int J Cardiol 2016; 225: 128-39.

17. Walen S, Katerberg B, Boomsma MF, et al. Safety, feasibility and patient reported outcome measures of outpatient treatment of pulmonary embolism. Thromb Res 2017; 156: 172-6.

18. Liang NL, Avgerinos ED, Singh MJ, et al. Systemic thrombolysis increases hemorrhagic stroke risk without survival benefit compared with catheter-directed intervention for the treatment of acute pulmonary embolism. J Vasc Surg Venous Lymph Disord 2017; 5: 171-6.

19. Jaff MR, MCMurtry MS, Archer SL, et al. Management of massive and submassive pulmonary embolism, iliofemoral deep vein thrombosis, and chronic thromboembolic pulmonary hypertension: a scientific statement from the American Heart Association. Circulation 2011; 123: 1788-830.

20. Lee KA, Cha A, Kumar MH, et al. Catheter-directed, ultrasound-assisted thrombolysis is a safe and effective treatment for pulmonary embolism, even in high-risk patients. J Vasc Surg Venous Lymph Disord 2017; 5: 165-70.

21. Kucher N, Boekstegers P, Müller OJ, et al. Randomized, controlled trial of ultrasound-assisted catheter-directed thrombolysis for acute intermediate-risk pulmonary embolism. Circulation 2014; 129: 479-86.

22. Piazza G, Hohlfelder B, Jaff MR, et al. A prospective, single-arm, multicenter trial of ultrasound-facilitated, catheter-directed, low-dose fibrinolysis for acute massive and submassive pulmonary embolism: the SEATTLE II Study. JACC Cardiovasc Interv 2015; 8: 1382-92.

23. Kuo WT, Gould MK, Louie JD, et al. Catheter-directed therapy for the treatment of massive pulmonary embolism: systematic review and meta-analysis of modern techniques. J Vasc Interv Radiol 2009; 20: 1431-40.

24. Dwarka SA, Schwartz SH, Smyth SH, et al. Bradyarrhythmias during use of the AngioJet system. J Vasc Interv Radiol 2006; 17: 1693-5.

25. Lee MS, Makkar RR, Singh V, et al. Pre-procedural administration of aminophylline does not prevent Angiojet rheolytic thrombectomy-induced bradyarrythmias. I Invasive Cardiol 2005; 17: 19-22.

Received: 25.01.2018, accepted: 2.03.2018. 